\title{
Responses of Patients with Disorders of Consciousness to Habit Stimulation: A Quantitative EEG Study
}

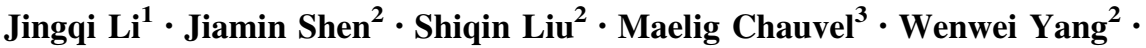 \\ Jian $\mathrm{Mei}^{2} \cdot \operatorname{Ling} \mathrm{Lei}^{2} \cdot \mathrm{Li} \mathrm{Wu}^{2} \cdot \mathrm{Jian} \mathrm{Gao}^{4} \cdot$ Yong Yang ${ }^{2}$
}

Received: 31 January 2018/Accepted: 13 June 2018/Published online: 17 July 2018

(C) The Author(s) 2018

\begin{abstract}
Whether habit stimulation is effective in DOC patient arousal has not been reported. In this paper, we analyzed the responses of DOC patients to habit stimulation. Nineteen DOC patients with alcohol consumption or smoking habits were recruited and 64-channel EEG signals were acquired both at the resting state and at three stimulation states. Wavelet transformation and nonlinear dynamics were used to extract the features of EEG signals and four brain lobes were selected to investigate the degree of EEG response to habit stimulation. Results showed that the highest degree of EEG response was from the callname stimulation, followed by habit and music stimulations. Significant differences in EEG wavelet energy and response coefficient were found both between habit and music stimulation, and between habit and call-name stimulation. These findings prove that habit stimulation induces relatively more intense EEG responses in DOC patients than music stimulation, suggesting that it may be a relevant additional method for eliciting patient arousal.
\end{abstract}

Jingqi Li and Jiamin Shen have contributed equally to this work.

Yong Yang

yyang@hdu.edu.cn

1 Ming Zhou Nao Kang Rehabilitation Hospital, Hangzhou 310000, China

2 College of Life Information Science and Instrument Engineering, Hangzhou Dianzi University, Hangzhou 310018, China

3 Paris Descartes University, 45 Rue des Saints-Peres, 75006 Paris, France

4 Rehabilitation Center, Wu Jing Hospital, Hangzhou 310051, China
Keywords EEG - Disorder of consciousness - Habit stimulation - Wavelet transformation · Nonlinear dynamics · Differential analysis

\section{Introduction}

Exploring an effective method to help the arousal of patients suffering from disorders of consciousness (DOCs) is an ongoing challenge [1, 2]. Sensitive stimulation treatments, including call-name and music stimulations, are the most commonly used methods [3, 4]. Habits such as alcohol consumption and smoking can arouse intense behavioral responses in normal individuals [5]. These habits are built on patients' specific and unique lifestyle behaviors. The detailed neurobiological mechanisms associated with habits remain unclear. It is still unknown whether habit stimulation can be applied to DOC patients, as few studies have compared patient responses to habit with those to other types of stimulation [6].

Clinical practice indicates that habit stimulation (e.g. smoking) arouses stronger behavioral responses than nonhabit stimulation (e.g. non-smoking) in DOC patients. For example, there was no response when vinegar or sauce was placed on the lips of patients who were alcoholics, but alcohol caused their lips to start moving in a drinking movement. Yet these behavioral responses may be subjective, and a quantitative study is needed to explain this phenomenon. In most cases, qualitative analyses such as neuropsychological scales [e.g. the Coma Recovery Scale Revised (CRS-R) score] are used to evaluate brain functions in the clinical setting. In order to thoroughly investigate these behavioral responses, more quantitative analytical methods are needed to accurately evaluate the brain responses. Diagnostic tools such as functional 
magnetic resonance imaging [7-9], positron emission tomography $[10,11]$, and electroencephalogram (EEG) analysis [12-17] have been used as auxiliary diagnostic methods to evaluate the state of consciousness. EEG has several advantages over the other methods, including low cost, safety, easy access, and convenience for bedside evaluation [18-21].

Generally, linear and nonlinear dynamic analysis methods are used to extract the features of EEG signals. In linear analysis, one of the common methods is timefrequency analysis, including variable-time-interval Fourier transformation, wavelet transformation, and WignerVille distribution. Among these, wavelet transformation is a typical time-frequency analysis with multi-resolution and multi-scale characteristics, which enables the conversion of a multi-signal from coarse to fine [22]. Wavelet transformation is a mature theory which has been widely used in the study of EEG signals [23]. Besides linear analysis, nonlinear dynamic analysis methods have also been widely used to extract features of EEG signals [23, 24]. Studies have shown that the human brain is a complex, multidimensional system, and the use of nonlinear dynamic analysis might accurately reflect brain states [25-27].

In this study, to investigate the response of DOC patients to habit stimulation, both linear and nonlinear dynamic analysis methods were applied to measure the response intensity of the EEG in DOC patients. In addition, topographic maps of the brain were plotted to evaluate the degree of response to stimulation in different regions.

\section{Materials and Methods}

\section{Patients}

A total of 19 patients with alcohol consumption or smoking habits were examined in this study, including 9 MCS (minimally conscious state) and 10 VS (vegetative state) cases assessed by neurologists based on the CRS-R. These patients were recruited from the Rehabilitation Center of Wu Jing Hospital in Zhejiang Province, China. Patients were out of the clinically acute phase, had been in a DOC state for $>1$ month, exhibited spontaneous breathing, and had no history of cardiopulmonary resuscitation or neurological disease. The inclusion criteria were as follows: (a) inability to follow commands; (b) inability to clearly express words; (c) inability to open eyes even with stimulation or achieve eye tracking, not due to paralysis; (d) inability to move arms and legs in a non-directed fashion; and (e) having a Glasgow coma scale [28] score $\leq 8$ points, and with a score for each item $<4-5-6-3-2-3$ according to the CRS-R [29]. If the total score was $\geq 8$, the patient was diagnosed as MCS; if it was $<8$, the patient was diagnosed as VS. The exclusion criteria were as follows: (a) drug interventions (which could affect the assessment of brain function) prior to data collection such as nerve-muscle blockers, depressants, or anesthetics; and (b) a coexisting disorder such as metabolic disease, poisoning, or shock that could affect brain activity.

The state of consciousness was diagnosed by experienced clinicians from Wu Jing Hospital using the CRS-R scale. The regional Review Board approved the use of human participants in this study. Family members of patients and the attending doctor gave consent for EEG acquisition. Patient information is summarized in Table 1.

\section{EEG Acquisition}

EEG signals were recorded in single-electrode channel mode with an Active Two EEG system (BioSemi, Amsterdam, Netherlands). Electrodes were placed over the entire head according to the 10-20 general international standard lead system. Signals were recorded from 64 channels; the left and right earlobes were used as references.

The EEG recording was initiated when the signal had been stable for at least $2 \mathrm{~min}$. Signals were digitized at a sampling rate of $256 \mathrm{~Hz}$, a bandwidth range from $0.5 \mathrm{~Hz}$ to $70 \mathrm{~Hz}$, and an electrode impedance $<5 \mathrm{~K} \Omega$.

In the music stimulation, a piece of Chinese classical music "Jasmine" was truncated into voice fragments and played for $90 \mathrm{~s}$. In the call-name stimulation, the patient's name was called by relatives for $90 \mathrm{~s}$. In the habit stimulation, patients were stimulated either by wiping alcohol on the lips for $36 \mathrm{~s}$ for alcoholic patients, or by introducing the smell of cigarette smoke for $36 \mathrm{~s}$ for smoking patients (Fig. 1). The entire process was repeated three times.

\section{EEG Signal Preprocessing}

A time-window of $12 \mathrm{~s}$ was truncated from the acquired EEG data for analysis. IIR filter, an EEGLAB processing tool (University of California San Diego), was used to remove interference from the $50-\mathrm{Hz}$ power frequency [30]. A wavelet soft threshold de-noising algorithm was applied to remove noise [31].

\section{Wavelet De-noising of EEG}

Studies have shown that the process of wavelet de-noising often uses a given threshold for the de-noising paradigm. The most reliable threshold method is calculated based on trials and errors [31]. There are several classical threshold methods: (a) The VisuShrink threshold, also known as the general threshold, was the first wavelet threshold de- 
Table 1 Patient information.

\begin{tabular}{|c|c|c|c|c|c|c|}
\hline \multirow[t]{2}{*}{ Disease status } & \multirow[t]{2}{*}{ Number of patients } & \multirow[t]{2}{*}{ CRS-R score } & \multirow[t]{2}{*}{ Average age (years) } & \multirow[t]{2}{*}{ Time after injury (months) } & \multicolumn{2}{|l|}{ Sex } \\
\hline & & & & & Male & Female \\
\hline MCS & 9 & $13.7 \pm 2.61$ & $39.3 \pm 11.9$ & $3.10 \pm 1.92$ & 8 & 1 \\
\hline VS & 10 & $6.2 \pm 1.90$ & $51.1 \pm 10.2$ & $4.05 \pm 1.38$ & 7 & 3 \\
\hline
\end{tabular}

CRS-R, Coma Recovery Scale - Revised; MCS, minimally conscious state; VS, vegetative state.

Fig. 1 Time-course of EEG acquisition.

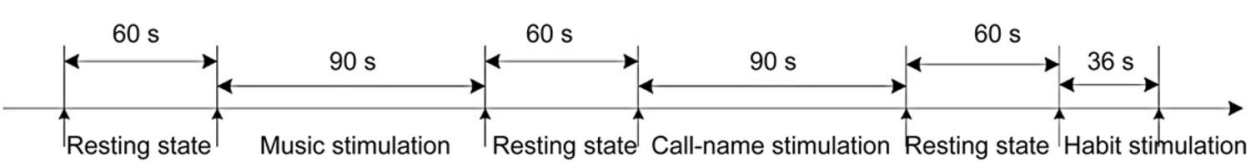

was complex, $0 \mathrm{~Hz}-32 \mathrm{~Hz}$ three-layer decomposition was taken as an example for the following wavelet decomposition corresponding band diagram (Fig. 2).

The energy value of a certain wavelet coefficient can be used to represent the energy value of a signal in a band. Since the wavelet function is an orthogonal basis function with energy conservation characteristics, the sum of the wavelet energy values of each band can represent the energy value of the signal, that is, the wavelet energy value of the signal:

$E_{n}=\sum_{k}\left|d_{l}^{j, n}\right|^{2}$

where both $n$ and $k$ are natural numbers and $d_{i}^{j, n}$ are wavelet packet coefficients.

Wavelet transformation was used to calculate EEG signal features under resting and stimulation states (music, habit, and call-name). The resting state was considered as the unified normalized standard of response coefficients. Therefore, the ratio between the wavelet transform value of the stimulation state and the wavelet transform value of the resting state was defined as the wavelet energy value. This ratio reflected the change of wavelet energy before and after stimulation. The EEG wavelet energy was calculated as:

$$
\begin{aligned}
& \text { Wavelet energy value (ratio) } \\
& =\text { wavelet energy value of stimulation state/ } \\
& \quad \text { wavelet energy value of resting state. }
\end{aligned}
$$

The signal was decomposed into eight layers, and a db3 wavelet base was chosen. As the eight-layer decomposition

Table 2 Comparison of four threshold estimation methods.

\begin{tabular}{lcccc}
\hline Threshold estimation method & VisuShrink & Sureshrink & Heursure & Minmax \\
\hline SNR & 42.541 & 72.890 & 72.890 & 50.997 \\
RSME & 1.217 & 0.037 & 0.0370 & 0.460 \\
\hline
\end{tabular}

SNR, signal-to-noise ratio; RSME, root-mean-square error. 


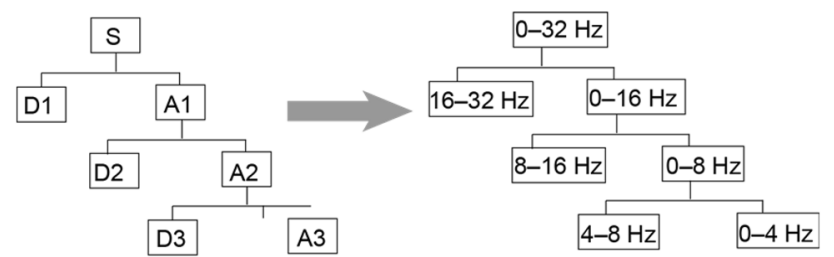

Fig. 2 Correspondence of wavelet decomposition band diagram.

\section{Nonlinear Dynamic Feature Extraction}

Correlation dimension, complexity, entropy, and Lyapunov exponents are common nonlinear features in EEG signal analysis. Of these, entropy is the most suitable because of its small dataset demand and high computation speed, whereas correlation dimension and Lyapunov exponent require large datasets and strict dimensions (features that are unsuitable for EEG analysis). Therefore, in this study, approximate entropy (ApEn) [32], sample entropy (SampEn) [33], and permutation entropy (PmEn) [34], were used to determine the patients' state of consciousness.

A nonlinear dynamic method was used to calculate EEG signal features (ApEn, SampEn, and PmEn) under the resting and stimulation states (music, habit, and call-name) [7]. During feature extraction, ApEn, SampEn, and PmEn were computed for the EEG data in each time-window (12 s signal truncated from the EEG data). For each nonlinear characteristic, the mean of the values from all time-windows was considered as a feature of the EEG data. The feature value was extracted and calculated from each channel. The means of single features were the average value of all 64 channel features of the EEG data.

The resting state was considered as the unified normalized standard of response coefficients (Rc). Therefore, the ratio between the nonlinear dynamic feature of the stimulation state and the nonlinear dynamic feature of the resting state was defined as the Rc. Rc values are the EEG nonlinear dynamic feature response coefficients of the stimulation value, which reflect the changes of a nonlinear dynamic feature before and after stimulation. The Rc values were calculated as:

Rc value (ratio) = feature of stimulation state / feature of resting state

$\mathrm{RcA}, \mathrm{RcS}$, and RcP refer to the Rc values for the three features ApEn, SampEn, and PmEn, respectively.

\section{Statistical Analysis}

Wavelet energy and Rc values were analyzed using the paired-samples $t$-test, independent-sample $t$-test, and oneway ANOVA, using SPSS v.19 software (SPSS Inc., Chicago, IL). $P<0.05$ was considered statistically significant.
Table 3 Comparison of wavelet energy values in different stimulation states.

\begin{tabular}{llll}
\hline Stimulation & Total $(\mathrm{MCS}+\mathrm{VS})$ & MCS & VS \\
\hline Music & $1.124 \pm 0.147$ & $1.139 \pm 0.158$ & $1.107 \pm 0.116$ \\
Habit & $1.346 \pm 0.198$ & $1.391 \pm 0.215$ & $1.296 \pm 0.115$ \\
Call-name & $1.423 \pm 0.314$ & $1.489 \pm 0.343$ & $1.349 \pm 0.128$ \\
\hline
\end{tabular}

\section{Results}

\section{EEG Wavelet Energy Values for MCS and VS Under Different Stimulations}

We first investigated the degree of EEG response to various kinds of stimulation by comparing the differences in wavelet energy. The highest wavelet energy of the cases (including MCS and VS) was for call-name stimulation, followed by habit and music stimulation (Table 3).

To verify the differences among the three stimulations, statistical analysis of the wavelet energy values between the different stimulations were performed. First, differences were analyzed between habit and music stimulation, then between habit and call-name stimulation, using the paired-samples $t$-test. In all cases, there were significant differences in the wavelet energy both for habit versus music ( $\mathrm{P}=0.0065)$ and for habit versus call-name stimulation $(P=0.0089)$. In MCS cases, the wavelet energy differed significantly $(P=0.0074)$ between habit and music, but not between habit and call-name stimulation. However, the VS cases showed no significant differences in wavelet energy either between habit and music, or between habit and call-name stimulation (Fig. 3).

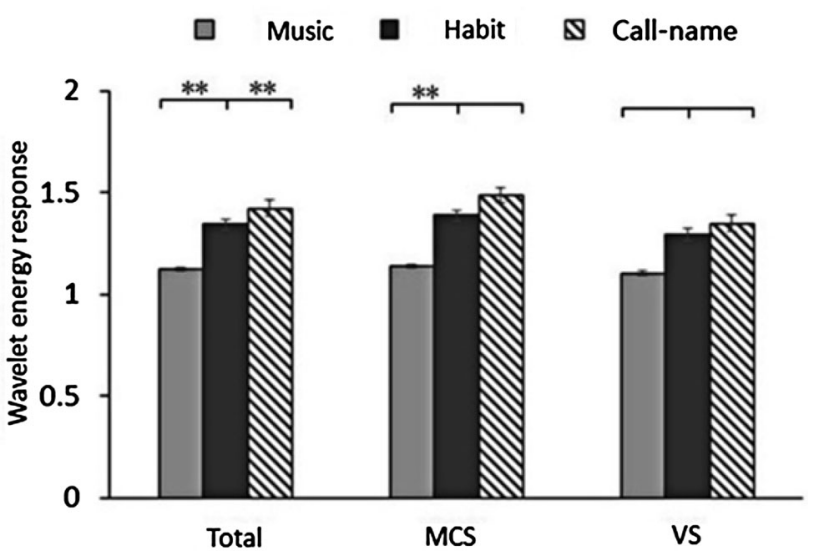

Fig. 3 Difference of wavelet energy values in total cases $(n=19)$, MCS cases $(n=9)$, and VS cases $(n=10)$ in the three stimulations (music, habit, and call-name) (error bars, 95\% confidence intervals; $* * P<0.01)$. 
Further investigations of the differences of wavelet energy values between VS and MCS cases for the three stimulations were performed using the independent-samples $t$-test. There was a significant difference in these values for habit $(P=0.023)$ and call-name stimulation $(P=0.016)$ between MCS and VS cases, but not for music stimulation (Fig. 4).

\section{EEG Re Values for MCS and VS Under Different Stimulations}

We then investigated the degree of EEG response to various kinds of stimulation by comparing the nonlinear entropy values in DOC patients. The Rc values (RcA, RcS, and $\mathrm{RcP}$ ) of the three different stimulations are shown in Table 4. The results indicated that the highest $\mathrm{Rc}$ values

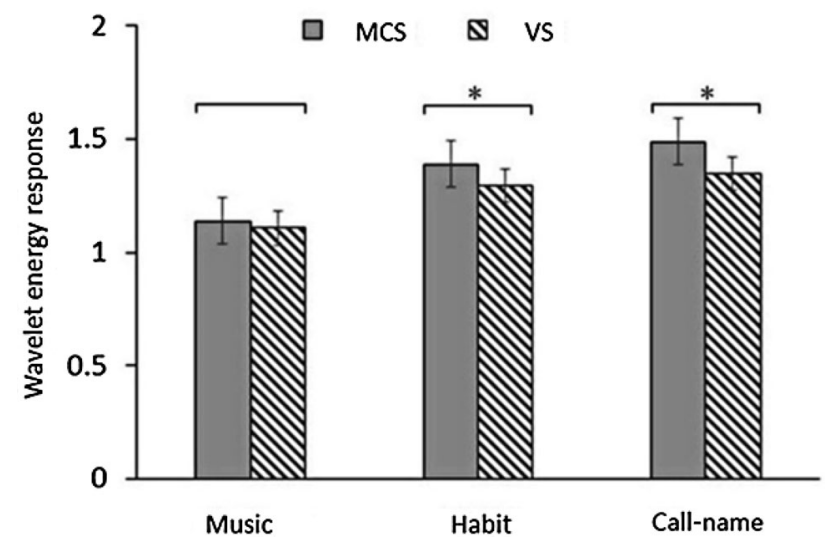

Fig. 4 Analysis of EEG wavelet energy value differences between VS and MCS cases for the three stimulations (error bars, 95\% confidence intervals; $* P<0.05)$.

Table 4 EEG entropy values in different stimulation states.

\begin{tabular}{|c|c|c|c|}
\hline \multirow[t]{2}{*}{ Sample } & \multicolumn{3}{|l|}{ Rc values } \\
\hline & $\mathrm{RcA}$ & $\mathrm{RcS}$ & $\mathrm{RcP}$ \\
\hline \multicolumn{4}{|c|}{ Total (MCS+VS) } \\
\hline Music & $1.044 \pm 0.13$ & $1.071 \pm 0.12$ & $1.004 \pm 0.01$ \\
\hline Habit & $1.262 \pm 0.05$ & $1.234 \pm 0.09$ & $1.254 \pm 0.31$ \\
\hline Call-name & $1.446 \pm 0.24$ & $1.254 \pm 0.21$ & $1.394 \pm 0.32$ \\
\hline \multicolumn{4}{|l|}{ MCS } \\
\hline Music & $1.025 \pm 0.15$ & $1.067 \pm 0.15$ & $1.004 \pm 0.02$ \\
\hline Habit & $1.413 \pm 0.07$ & $1.377 \pm 0.13$ & $1.413 \pm 0.23$ \\
\hline Call-name & $1.668 \pm 0.21$ & $1.644 \pm 0.19$ & $1.602 \pm 0.28$ \\
\hline \multicolumn{4}{|l|}{ VS } \\
\hline Music & $1.061 \pm 0.13$ & $1.074 \pm 0.08$ & $1.003 \pm 0.004$ \\
\hline Habit & $1.127 \pm 0.09$ & $1.105 \pm 0.06$ & $1.111 \pm 0.36$ \\
\hline Call-name & $1.247 \pm 0.17$ & $1.211 \pm 0.15$ & $1.206 \pm 0.24$ \\
\hline
\end{tabular}

among all the cases corresponded to call-name stimulation, followed by habit and music stimulation.

In order to verify the statistical differences between the three stimulations, statistical analysis of the Rc values for habit versus music, and for habit versus call-name stimulation was performed using the paired-samples $t$-test. In
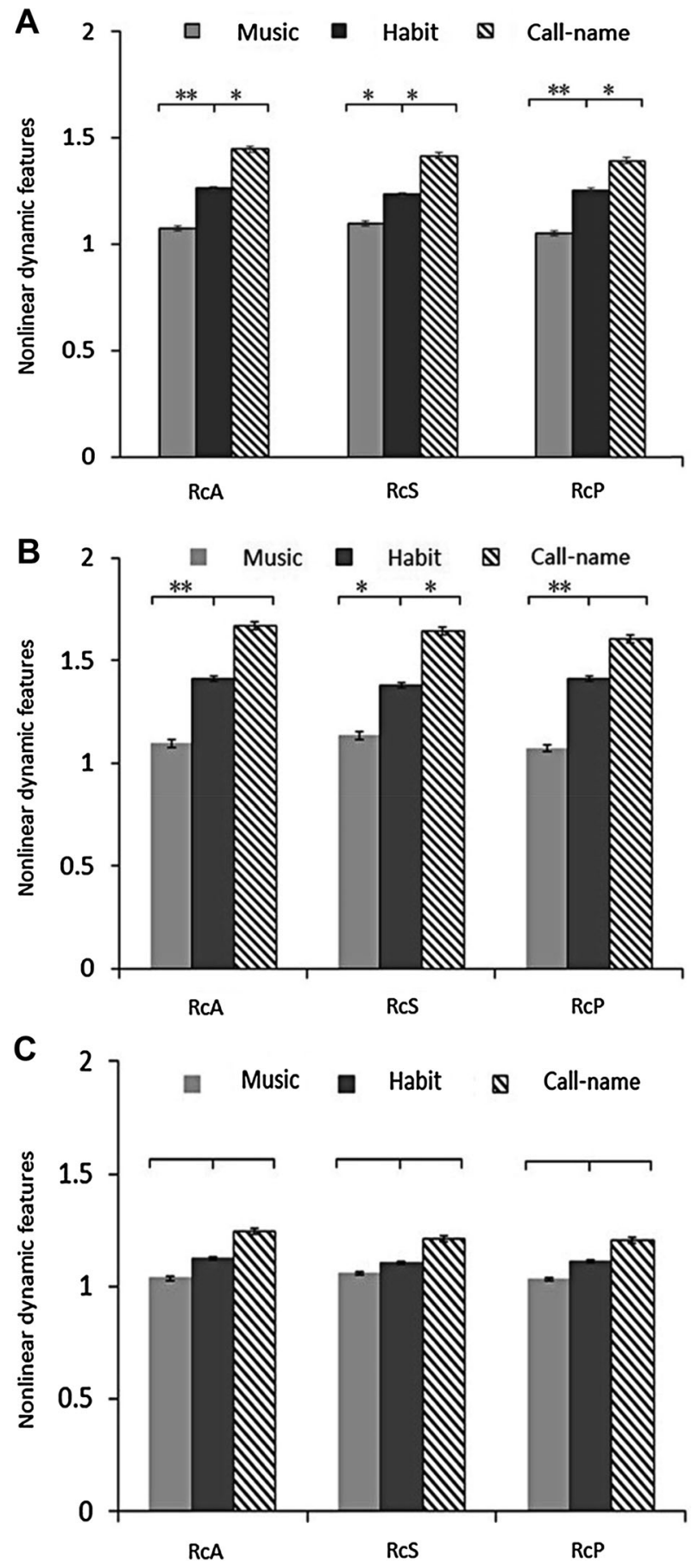

Fig. 5 Rc values of A the total patient population ( $n=19)$, B MCS cases $(n=9)$, and $\mathbf{C}$ VS cases $(n=10)$ in response to the three stimulations (error bars, $95 \%$ confidence intervals; $* P<0.05$, $* * P<0.01)$. 
Table 5 Analysis of EEG Rc differences ( $P$ values) between VS and MCS for the three stimulations.

\begin{tabular}{llll}
\hline Feature value & Music & Habit & Call-name \\
\hline RcA & 0.177 & 0.006 & 0.007 \\
RcS & 0.894 & 0.004 & 0.001 \\
RcP & 0.871 & 0.032 & 0.003 \\
\hline
\end{tabular}

all cases, there were significant differences in the three Rc values between habit versus call-name stimulation (RcA, $P=0.029$; RcS, $P=0.034$; RcP, $P=0.041)$. Moreover, two Rc values (RcA and RcP) remarkably differed between habit and music stimulations $(P=0.0082, P=0.0096)$ (Fig. 5A). In MCS cases, there were significant differences in the three Rc values between habit and music stimulations (RcA, $P=0.0097 ; \quad \mathrm{RcS}, \quad P=0.037 ; \quad \mathrm{RcP}$, $P=0.0088)$; but only RcS differed $(P=0.038)$ between habit and call-name stimulations (Fig. 5B). In VS cases, there were no differences in the three Rc values either between habit and music or between habit and call-name stimulations (Fig. 5C).

Further comparison of the Rc values between VS and MCS cases was performed using the independent-samples $t$-test. RcA and RcS differed significantly (RcA, $P=0.006$; $\mathrm{RcS}, P=0.004)$ between MCS and VS cases for habit stimulation; all three differed significantly (RcA, $P=0.007$; RcS, $P=0.001$; RcP, $P=0.003$ ) between MCS and VS cases for call-name stimulation; and none was significantly different between MCS and VS cases for music stimulation (Table 5).

\section{EEG Response in Different Brain Lobes Under Different Stimulations}

Since different stimulations may activate distinct brain areas, we finally investigated the degree of EEG responses in the frontal, temporal, parietal, and occipital lobes at different regions elicited by various types of stimulation.

Among the nonlinear EEG features (ApEn, SampEn, and PmEn), PmEn measures the complexity of a signal, with the advantages of being simple, fast, and having a strong anti-noise property. Therefore, PmEn was used to measure the nonlinear dynamic feature responses of the different areas. The differences of entropy values in the different areas during the three stimulations were evaluated using one-way ANOVA.

The resulting EEG responses in the different lobes under different stimulations are shown in Table 6. During habit stimulation, the RcP values in the frontal lobe were significantly higher than those in any other areas in VS cases $(P<0.05)$, MCS cases $(P<0.01)$, and VS + MCS cases $(P<0.01)$. During music stimulation, the RcP values in the temporal lobe were significantly higher $(P<0.05)$ than those in any other areas for VS + MCS and VS cases, but not for MCS cases. During call-name stimulation, the $\mathrm{RcP}$ values in the temporal lobe were significantly higher than those in any other areas for VS + MCS $(P<0.05)$, VS $(P<0.05)$, and MCS $(P<0.01)$.

To visualize the results of brain responses under different stimulations, we used the topographic map visualization method. Three VS cases and 4 MCS cases were chosen to plot the maps using the mean PmEn (Fig. 6). These patients did not suffer from traumatic brain injury, but from other conditions such as subarachnoid hemorrhage or diffuse axonal injury. Indeed, in these cases,
Table 6 EEG entropy values in different stimulation states.

\begin{tabular}{|c|c|c|c|c|}
\hline \multirow[t]{2}{*}{ Sample } & \multicolumn{4}{|l|}{ Brain area } \\
\hline & Frontal lobe & Temporal lobe & Parietal lobe & Occipital lobe \\
\hline \multicolumn{5}{|c|}{ Total $(\mathrm{MCS}+\mathrm{VS})$} \\
\hline Music & $0.986 \pm 0.097$ & $1.048 \pm 0.105^{*}$ & $1.003 \pm 0.079$ & $0.985 \pm 0.103$ \\
\hline Habit & $1.452 \pm 0.132 * *$ & $1.116 \pm 0.097$ & $1.179 \pm 0.099$ & $1.294 \pm 0.154$ \\
\hline Call-name & $1.378 \pm 0.112$ & $1.530 \pm 0.101 *$ & $1.315 \pm 0.101$ & $1.393 \pm 0.145$ \\
\hline \multicolumn{5}{|l|}{ MCS } \\
\hline Music & $1.014 \pm 0.059$ & $1.024 \pm 0.062$ & $1.005 \pm 0.050$ & $0.972 \pm 0.057$ \\
\hline Habit & $1.664 \pm 0.163 * *$ & $1.215 \pm 0.099$ & $1.280 \pm 0.104$ & $1.492 \pm 0.138$ \\
\hline Call-name & $1.601 \pm 0.068$ & $1.730 \pm 0.110^{* *}$ & $1.447 \pm 0.126$ & $1.631 \pm 0.162$ \\
\hline \multicolumn{5}{|l|}{ VS } \\
\hline Music & $0.973 \pm 0.122$ & $1.072 \pm 0.135^{*}$ & $0.967 \pm 0.101$ & $0.998 \pm 0.135$ \\
\hline Habit & $1.240 \pm 0.103 *$ & $1.018 \pm 0.100$ & $1.077 \pm 0.099$ & $1.096 \pm 0.165$ \\
\hline Call-name & $1.155 \pm 0.139$ & $1.331 \pm 0.092^{*}$ & $1.183 \pm 0.064$ & $1.154 \pm 0.099$ \\
\hline
\end{tabular}


Fig. 6 EEG topographic maps of the mean PmEn under the three stimulations. The colors reflect the intensity of the EEG response. MCS and VS results for music stimulation (upper panels), habit stimulation (middle panels), and call-name stimulation (lower panels).
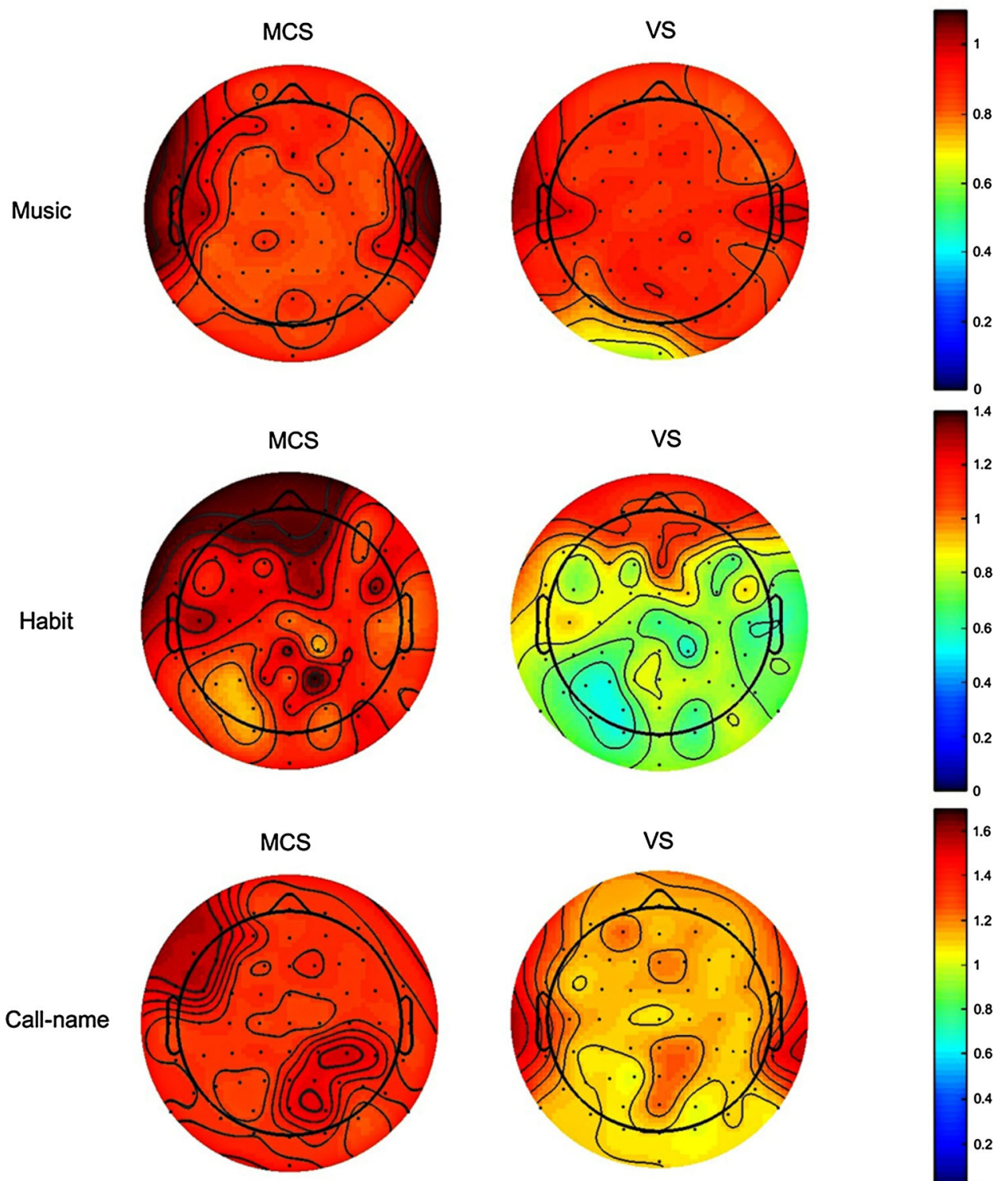

the brain integrity was conserved and the mapping was not influenced by injury of the lobes.

With the music stimulation, in both MCS and VS cases, the darkest area was located in the temporal cortex, denoting a significantly more intense EEG response than in other regions. Furthermore, the EEG responses in MCS cases were more intense than those of VS cases. The results of music stimulation showed that, in the selected cases, the temporal region was the most responsive.

With the habit stimulation, in both MCS and VS cases, the darkest area was located in the prefrontal region, which had significantly more intense EEG responses than other regions. The EEG responses of the MCS cases were more intense than those of the VS cases. The results of habit stimulation demonstrated that, for the selected cases, the frontal brain region was the most responsive area.
With the call-name stimulation, in the MCS cases, the darkest areas were in the left temporal and right occipital areas, which had significantly more intense EEG responses than other regions. In the VS cases with call-name stimulation, the darkest area was in the temporal area, which had significantly more intense EEG responses than other regions. The EEG responses of MCS cases were more intense than those of VS cases.

\section{Discussion}

In this study, using EEG wavelet transformation and nonlinear dynamics analysis, we studied the EEG responses of DOC patients under different stimulations to determine whether habit stimulation is clinically useful. 
We found that, with the three types of stimulation (music, habit, and call-name), the EEG response under habit stimulation was higher than that under music stimulation, but lower than that under call-name stimulation. In different consciousness states, the MCS response to habit stimulation was more pronounced than that for VS patients. These results provide preliminary evidence for the effectiveness of habit stimulation. Concerning the spatial distribution of EEG responses, the brain maps showed a more intense response in the frontal lobe during habit stimulation and in the temporal lobe during music and callname stimulation. These results may reflect positive neuralrelated activity evoked by the stimulations.

Results from current studies suggest that habit stimulation can arouse brain responses; however, the detailed neurobiological mechanism underlying habit remains unclear but could be involved in the addiction mechanism. Existing research shows that the mesolimbic dopamine system (MLDS) is the neurobiological basis of the addiction mechanism [35]. The MLDS is a pathway by which dopamine is carried from one area to another. Dopamine is the major molecule released by the brain's reward centers. Dopaminergic neurons are mainly located in the ventral tegmental area, and also have projections into several parts of the brain, including the nucleus accumbens, prefrontal cortex, hippocampi, and amygdala. The MLDS is the common neural pathway of the reward mechanism, and is involved in the physiological process of addiction [36]. In nicotine habit stimulation, the MLDS is thought to be the key site of its action. However, nicotine also increases the extracellular dopamine concentration by stimulating dopaminergic neurons. In alcohol habit stimulation, it has been reported that alcohol stimulates addiction, triggers dopamine release, enhances brain activity, and stimulates the reward system. Rose [37] proposed that nicotine and alcohol have positive reinforcement in the MLDS. The specific neural mechanisms involved in habit stimulation need to be further clarified using imaging techniques such as fMRI combined with EEG, as well as neuropsychological scale methods. Future investigations need larger samples.

Acknowledgements This work was supported by the National Natural Science Foundation of China (81671038).

\section{Compliance with Ethical Standards}

Conflict of interest The authors claim no conflict of interest.

Open Access This article is distributed under the terms of the Creative Commons Attribution 4.0 International License (http:// creativecommons.org/licenses/by/4.0/), which permits unrestricted use, distribution, and reproduction in any medium, provided you give appropriate credit to the original author(s) and the source, provide a link to the Creative Commons license, and indicate if changes were made.

\section{References}

1. Jox RJ, Bernat JL, Laureys S. Disorders of consciousness: responding to requests for novel diagnostic and therapeutic interventions. Lancet Neurol 2012, 11: 841.

2. Giacino JT, Ashwal S, Childs N, Cranford R, Jennett B, Katz DI, et al. The minimally conscious state definition and diagnostic criteria. Neurology 2002, 58.

3. Abbate C, Trimarchi PD, Basile I, Mazzucchi A, Devalle G. Sensory stimulation for patients with disorders of consciousness: from stimulation to rehabilitation. Front Hum Neurosci 2014, 8: 616.

4. Qin P, Di H, Yan X, Yu S, Yu D, Laureys S, et al. Mismatch negativity to the patient's own name in chronic disorders of consciousness. Neurosci Lett 2008, 448: 24-28.

5. McClernon FJ, Kozink RV, Lutz AM, Rose JE. 24-h smoking abstinence potentiates fMRI-BOLD activation to smoking cues in cerebral cortex and dorsal striatum. Psychopharmacology (Berl) 2009, 204: 25-35.

6. Nikulin VV, Linkenkaer-Hansen K, Nolte G, Lemm S, Müller KR, Ilmoniemi RJ, et al. A novel mechanism for evoked responses in the human brain. Eur J Neurosci 2007, 25: 3146-3154.

7. Di HB, Yu SM, Weng XC, Laureys S, Yu D, Li JQ, et al. Cerebral response to patient's own name in the vegetative and minimally conscious states. Neurology 2007, 20: 895-899.

8. Vanhaudenhuyse A, Noirhomme Q, Tshibanda LJF, Bruno MA, Boveroux P, Schnakers C, et al. Default network connectivity reflects the level of consciousness in non-communicative braindamaged patients. Brain 2010, 133: 161-171.

9. Fernandez-Espejo D, Bekinschtein T, Monti MM, Pickard JD, Junque C, Coleman MR, et al. Diffusion weighted imaging distinguishes the vegetative state from the minimally conscious state. Neuroimage 2011, 54: 103-112.

10. Boly M, Faymonville ME, Schnakers C, Peigneux P, Lambermont B, Phillips C, et al. Perception of pain in the minimally conscious state with PET activation: an observational study. Lancet Neurol 2008, 7: 1013-1020.

11. Laureys S, Goldman S, Phillips C, Van Bogaert P, Aerts J, Luxen A, et al. Impaired effective cortical connectivity in vegetative state: preliminary investigation using PET. Neuroimage 1999, 9: 377-382.

12. Ward LM, Mangia AL, Pirini M, Simoncini L, Cappello A. A feasibility study of an improved procedure for using EEG to detect brain responses to imagery instruction in patients with disorders of consciousness. PLoS One 2014, 9: e99289.

13. Faugeras F, Rohaut B, Weiss N, Bekinschtein TA, Galanaud D, Puybasset L, et al. Probing consciousness with event-related potentials in the vegetative state. Neurology 2011, 77: 264-268.

14. Fingelkurts AA, Fingelkurts AA, Bagnato S, Boccagni C, Galardi G. EEG oscillatory states as neuro-phenomenology of consciousness as revealed from patients in vegetative and minimally conscious states. Conscious Cogn 2012, 21: 149-169.

15. Goldfine AM, Victor JD, Conte MM, Bardin JC, Schiff ND. Determination of awareness in patients with severe brain injury using EEG power spectral analysis. Clin Neurophysiol 2011, 122: $2157-2168$.

16. Lehembre R, Marie-Aurélie B, Vanhaudenhuyse A, Chatelle C, Cologan V, Leclercq Y, et al. Resting-state EEG study of comatose patients: a connectivity and frequency analysis to find differences between vegetative and minimally conscious states. Funct Neurol 2012, 27: 41-47.

17. Rosanova M, Gosseries O, Casarotto S, Boly M, Casali AG, Bruno MA, et al. Recovery of cortical effective connectivity and recovery of consciousness in vegetative patients. Brain 2012, 135: $1308-1320$. 
18. Cruse D, Chennu S, Chatelle C, Bekinschtein TA, FernándezEspejo D, Pickard JD, et al. Bedside detection of awareness in the vegetative state: a cohort study. Lancet 2011, 378: 2088-2094.

19. Fellinger R, Klimesch W, Schnakers C, Perrin F, Freunberger R, Gruber W, et al. Cognitive processes in disorders of consciousness as revealed by EEG time-frequency analyses. Clin Neurophysiol 2011, 122: 2177-2184.

20. Li Y, Liu XP, Ling XH, Li JQ, Yang WW, Zhang DK, et al. Mapping brain injury with symmetrical-channels' EEG signal analysis-a pilot study. Sci Rep 2014, 4: 5023.

21. Jin B, So NK, Wang S. Advances of intracranial electroencephalography in localizing the epileptogenic zone. Neurosci Bull 2016, 32: 493-500.

22. Fraiwan L, Lweesy K, Khasawneh N, Wenz H, Dickhaus H. Automated sleep stage identification system based on timefrequency analysis of a single EEG channel and random forest classifier. Comput Methods Progr Biomed 2012, 108: 10-19.

23. Mirzaei A, Ayatollahi A, Gifani P, Salehi L. EEG analysis based on wavelet-spectral entropy for epileptic seizures detection. IEEE. 2010. https://doi.org/10.1109/BMEI.2010.5639894.

24. Gosseries O, Schnakers C, Ledoux D, Vanhaudenhuyse A, Bruno MA, Demertzi A, et al. Automated EEG entropy measurements in coma, vegetative state/unresponsive wakefulness syndrome and minimally conscious state. Funct Neurol 2011, 26: 25-30.

25. Stam CJ. Nonlinear dynamical analysis of EEG and MEG: review of an emerging field. Clin Neurophysiol 2005, 116: 2266-2301.

26. Mikšovský J, Raidl A. Testing for nonlinearity in European climatic time series by the method of surrogate data. Theor Appl Climatol 2005, 83: 21-33.

27. Kang JH, Chung YG, Kim SP. An efficient detection of epileptic seizure by differentiation and spectral analysis of electroencephalograms. Comput Biol Med 2015, 66: 352-356.
28. Teasdale G, Jennett B. Assessment of coma and impaired consciousness. A practical scale. Lancet 1974, 2: 81-84.

29. Giacino JT, Kalmar K, Whyte J. The JFK Coma Recovery ScaleRevised: Measurement characteristics and diagnostic utility 11 No commercial party having a direct financial interest in the results of the research supporting this article has or will confer a benefit upon the authors or upon any organization with which the authors are associated. Arch Phys Med Rehabilitation 2004, 85: 2020-2029.

30. Delorme A, Makeig S. EEGLAB: an open source toolbox for analysis of single-trial EEG dynamics including independent component analysis. J Neurosci Methods 2004, 134: 9-21.

31. Yuan FL, Luo ZZ. The EEG de-noising research based on wavelet and Hilbert Transform Method 2012: 361-365.

32. Pincus S. Approximate entropy (ApEn) as a complexity measure. Chaos 1995, 5: 110-117.

33. Di HB, Yu SM, Weng XC, Laureys S, Yu D, Li JQ, et al. Cerebral response to patient's own name in the vegetative and minimally conscious states. Neurology 2007, 68: 895-899.

34. Pincus SM. Approximate entropy as a measure of system complexity. Proc Natl Acad Sci U S A 1991, 88: 2297-2301.

35. Cao JP, Wang HJ, Li L, Zhang SM. The effects of morphine treatment on the NCAM and its signaling in the MLDS of rats. Drug Chem Toxicol 2016, 39: 418-423.

36. Wallis JD. Cross-species studies of orbitofrontal cortex and value-based decision-making. Nat Neurosci 2011, 15: 13.

37. Rose JE, Behm FM, Westman EC, Mathew RJ, London ED, Hawk TC, et al. PET studies of the influences of nicotine on neural systems in cigarette smoker. Am J Psychiatry 2003, 160: 323-333. 